P181 (continued)

readings and videos to complete the training and most $(11 ; 79 \%)$ felt the combination of both was most effective for learning. After completing the training, 11 (79\%) of respondents felt prepared to collect data using ASA24-2016.

Conclusions and Implications: Respondents had positive feedback for the content, reading level, appearance and design, and activities of the training. Results from this study suggest that the training is effective in preparing EFNEP nutrition educators to collect 24-hour dietary recall data using ASA24-2016; although potential bias due to self-selection and non-response is a concern. The feedback provided will inform future improvements to the manual.

Funding: USDA

\section{P182 Outcome Evaluation of a School-Based Intervention Implemented by Maine SNAP-Ed}

Brenda Wolford, MS, RD, brenda.wolford@altarum.org, Altarum Institute, 4 Milk Street, 3rd Floor, Portland, ME 04101; Rebecca Harnik, MS, Altarum Institute; Brent Walker, MS, RD; Margaret Wilkin, MPH; Patricia Dushuttle, MA, State of Maine DHHS Office for Family Independence; Elizabeth Pratt, MPH, University of New England; Pamela Bruno, MPH; Kira Rodriguez, MHS

Objective: To compare changes over time in fruit and vegetable consumption of students receiving a multi-level nutrition education intervention.

Theory, Prior Research, Rationale: Maine SNAP-Ed delivers evidence-based interventions in low-income communities supported by policy, systems and environmental (PSE) change strategies. Maine's SNAP-Ed implementing agency, the University of New England, selected Pick a Better Snack (PABS) for use in elementary schools. PABS, developed by the Iowa Nutrition Network, was previously evaluated and shown to significantly impact child fruit and vegetable consumption.

Description: PABS offers monthly lessons, take-home materials for parents and leave-behind classroom lessons. Supported by PSE strategies, PABS focuses on improving dietary intake and promoting physical activity. Target audience were second and third grade students and their parents/caregivers in eligible schools receiving the intervention.

Evaluation: Altarum conducted an evaluation of 222 families using pre/post-tests, and UNE conducted a postonly evaluation of classroom teachers.

Conclusions and Implications: Following the intervention, the evaluation revealed increases in frequency of children asking for fruits and vegetables and eating multiple kinds of fruits; and in parents/caregivers offering fruits and vegetables. After indirect education, parents/caregivers increased their own fruit and vegetable consumption. Take-home materials had a significant association with healthy eating; the child's consumption of fruits and vegetables increased if the parent reported using the information sent home. These findings reinforce the importance of extending nutrition education to teachers and parents. Additionally, PSE work in settings where PABS has been offered may help sustain positive changes. PABS appears to be an effective intervention to improve dietary intake among low-income families in Maine.

Funding: Supplemental Nutrition Assistance Program Education

\section{P183 Outcome Evaluation of Maine SNAP-Ed's Print and Digital Social Marketing Campaign}

Brent Walker, MS, RD, brent.walker@altarum.org, Altarum Institute, 4 Milk Street, 3rd Floor, Portland, ME 04101; Brenda Wolford, MS, $R D$, Altarum Institute; Patricia Dushuttle, MA, State of Maine DHHS Office for Family Independence

Objective: To determine effectiveness of reaching Maine SNAP-Ed's target audience with social marketing messages delivered through print and digital channels.

Theory, Prior Research, Rationale: USDA's Food and Nutrition Service (FNS) encourages the use of social marketing campaigns as a way to enhance nutrition education in SNAP-Ed. Successful campaigns are comprehensive in scope and provide education through multiple channels.

Description: In 2015, Maine SNAP-Ed launched its campaign, called Shop, Cook, Eat Healthy and on a Budget, to influence health-related behaviors of individuals and communities while building upon direct nutrition education efforts. The campaign integrated traditional print materials (direct mail postcards, posters, flyers), with a robust online presence (targeted online ads, social media, landing page). Target audience were SNAP-eligible women with children in Maine.

Evaluation: Altarum conducted a follow-up randomized phone survey of 600 SNAP recipients statewide using measures from the SNAP-Ed Evaluation Framework to assess campaign exposure. Awareness of the campaign increased significantly from the previous year ( $63 \%$ vs. $49 \%)$, and women with children reported higher levels of exposure to messages compared to other respondents ( $81 \%$ vs. $56 \%)$, indicating that messages are resonating with the target audience. While print materials were the most commonly recalled among all respondents, women with children had higher levels of recall of the digital campaign compared to others. Exposure to the campaign led many respondents to report tying out a new behavior such as making healthier food choices (32\%).

Conclusions and Implications: Evaluation results indicate that Maine SNAP-Ed has designed a social marketing campaign that reaches a large segment of its target audience through print and digital channels.

Funding: Supplemental Nutrition Assistance Program Education 